\title{
AN INVERSE KINEMATIC ALGORITHM FOR THE HUMAN LEG
}

\author{
Sebastian GŁowiński, Tomasz KrZYŻYŃski \\ Koszalin University of Technology, Faculty of Technology and Education, Koszalin, Poland \\ e-mail: sebastian.glowinski@tu.koszalin.pl; tomasz.krzyzynski@tu.koszalin.pl
}

\begin{abstract}
In this article, a 3-link kinematic model of a human leg is defined and analyzed with focus on optimizing the manipulability. The forward kinematics for the leg is used to define quantitative measures of the manipulability and workspace in a sagittal plane. Analytical results for different manipulability indices are derived. Using numerical optimization in Matlab package, the manipulability measure is optimized under different constraints. The range of motion and joint comfort zones of every joint is defined. The algorithm for redundant chain, based on analytical equations, is proposed in inverse kinematics.
\end{abstract}

Keywords: inverse kinematics, human leg, optimization

\section{Introduction}

The inverse kinematics of a human leg is the mapping that, given a goal position, calculates a set of joint positions so as to place the human leg effector (e.g. toe) in the specified goal. It is very important in the rehabilitation process. In this work, we present the main concerns on finding an inverse kinematics algorithm for a 3 link kinematic leg in plane. The work is divided into two parts: the first one, describing the analytical method for solving inverse kinematics, and the second one about the numerical method by using Matlab package. Inverse kinematics algorithms have been an issue to focus on since the first robots vave been built. The most popular methods have been the analytical ones (Parker et al., 1989), but an exact solution does not always exist. Therefore, sometimes alternative methods are used as interval methods (Rao et al., 1998), based on distances (Porta et al., 2006), genetic algorithms (Parker et al., 1989), or based on neural networks (Tejomurtula and Kak, 1999). This paper presents a numerical approach to solve the problem of multiple inverse kinematic solutions of a 3-link redundant manipulators (like the model of a human leg) to find a single optimum solution. A simulation model of this approach has been developed and computer simulations have been conducted by using Matlab package. The movement of the hip joint has not been implemented in the proposed algorithm, whereas it can be significant and should be included in the future studies. For people with injured spinal cord, the most important is verticalization. The authors treated the hip as stationary. Future work can be done in this direction by extending this approach to the 3 dimensional model with an increased number of links and joints. The described approach is simple and very fast in nature while solving inverse kinematics in comparison with genetic algorithms.

\section{Kinematic model of the leg}

The human structure is constituted by a skeleton and a number of muscles, which are collectively called the human musculoskeletal system. The human skeleton is a framework that consists of more than 200 bones $(\mathrm{Gu}, 2013)$. The movements of parts of the human body are presented in Fig. 1. Circumduction is a circular movement that combines flexion/extension, abduction/adduction. 

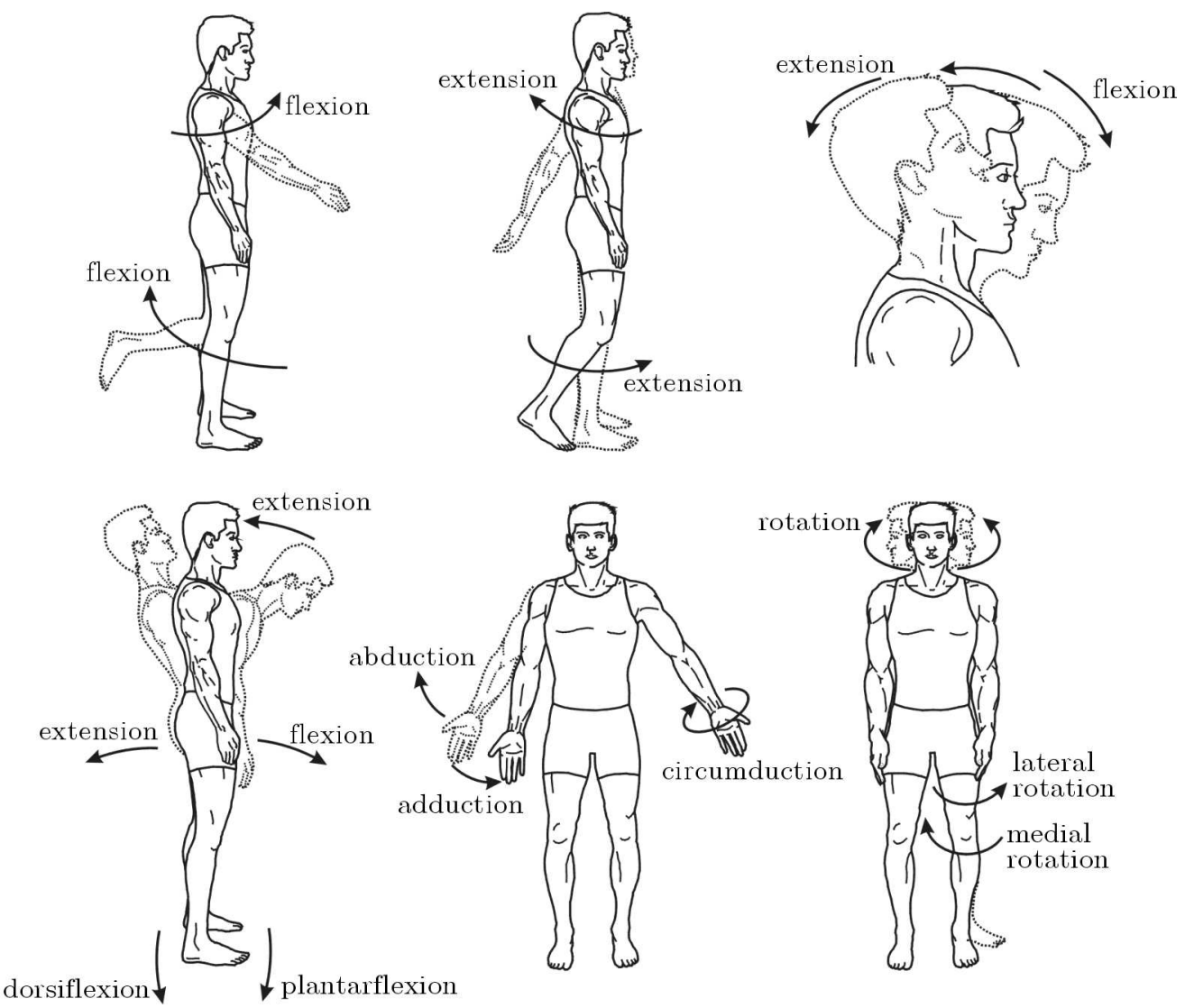

Fig. 1. Human joint movement. Reproduced from [9]

\subsection{Model of the human leg}

The proposed kinematic human leg model, in a sagittal plane, which passes from anterior to posterior, dividing the body into right and left halves, is presented in Fig. 2. The system of articulated links connected by rotatory joints are adopted to illustrate the human leg in this study. The leg is described as a system consisting of three segments, thigh, shank and foot as the length between ankle and metatarsal. The leg can be represented topologically using a kinematic chain structure in which links represent leg segments. The proposed model is kinematically redundant, because it possesses more degrees of freedom than those required to place the effector in a specified goal. To obtain the kinematic parameters, we make the following assumptions:

- The leg base is located at the origin $C\left(x_{C}, y_{C}\right)$ (hip joint), the knee joint $G\left(x_{G}, y_{G}\right)$, the ankle joint $K\left(x_{K}, y_{K}\right)$ and end effector (metatarsal) $O\left(x_{O}, y_{O}\right)$, respectively;

- Lengths of links are calculated as a function of human height $H[\mathrm{~m}]$, thigh bone $l_{t}=0.2450 \mathrm{H}$, shank $l_{l}=0.2460 \mathrm{H}$, length from ankle to metatarsal $l_{f}=0.0577 \mathrm{H}$;

- Joints are revolute and the limitations $\theta_{h}, \theta_{k}, \theta_{a}$ are known;

- The initial joint angles $\theta_{h}, \theta_{k}, \theta_{a}$ are known;

- The coordinates of the goal are given.

\subsection{The range of motion and comfort zone}

The range of motion (ROM) of every joint is determined not only by the mechanical structure, but also by many human factors, such as the use, body build, gender, health condition, age and 
(a)

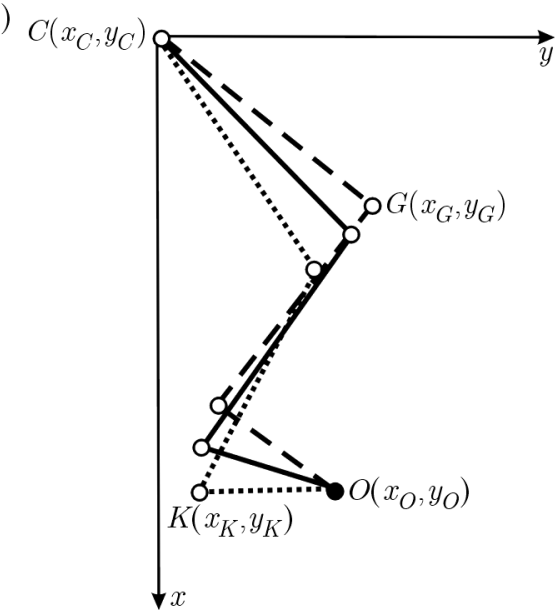

(b)

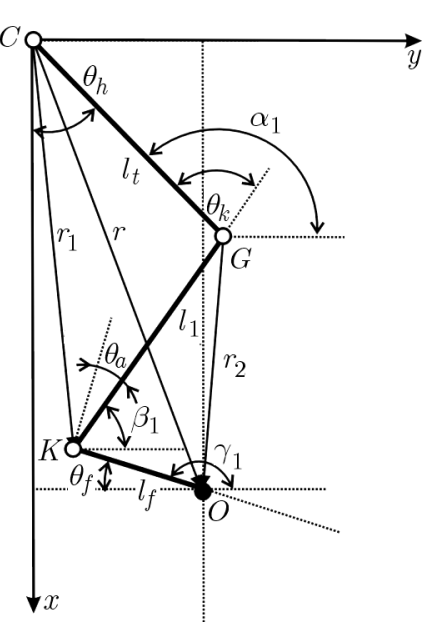

Fig. 2. Model of the human leg in a sagittal plane

many other factors (Chaffin and Andersson, 1991). The comfort zone (CZ) of each joint of the human leg, should be a subset of the corresponding joint ROM. Table 1 lists those average joint ROM's and the comfort zones just as a reference. The appropriate value of the upper-lower limit of each comfort zone is calculated by $0.35 \times$ the upper or lower limit of the corresponding human leg joint ROM. All the ROM data in the table are referred to the literature of biomechanics and kinesiology as average ranges (Tejomurtula and Kak, 1999). The comfort zone of each joint is determined by $35 \%$ of the ROM values, and the comfort center $\theta_{i}^{C}$ for each joint angle $\theta_{i}$ can be obtained as

$$
\theta_{i}^{C}=\frac{1}{2}\left(\theta_{i C Z}^{u}-\theta_{i C Z}^{l}\right)+\theta_{i}^{h}
$$

where $\theta_{i C Z}^{u}$ and $\theta_{i C Z}^{l}$ are the first and second angles of the comfort zone, respectively, for the corresponding joint $i$, and $\theta_{i}^{h}$ is the $i$-th joint home position. For example, $\theta_{a}$ is the joint angle of ankle plantarflexion/dorsiflexion with its home position $\theta_{a}^{h}=0^{\circ}$ (knee neural $0^{\circ}$ ). According to Table $1, \theta_{a C Z}^{u}=13.30^{\circ}$ and $\theta_{a C Z}^{l}=-12,25^{\circ}$. The ankle comfort zone plantarflexion/dorsiflexion joint can be calculated as $\theta_{a}^{C}=\left(13.30^{\circ}+12.25^{\circ}\right) / 2-0^{\circ}=12.78^{\circ}$. This calculation can be useful to set up the joint comfort optimization criterion in trajectory generation.

Table 1. The average joint ROM's and joint comfort zones

\begin{tabular}{|l|c|c|c|}
\hline \multicolumn{1}{|c|}{$\begin{array}{c}\text { Joint } \\
\text { mobility }\end{array}$} & $\begin{array}{c}\text { ROM } \\
{[\mathrm{deg}]}\end{array}$ & $\begin{array}{c}\text { Comfort zone } \\
{[\mathrm{deg}]}\end{array}$ & $\begin{array}{c}\text { Conditions } \\
\text { when }\end{array}$ \\
\hline \hline \multirow{2}{*}{$\begin{array}{l}\text { Hip } \\
\text { flexion/extension }\end{array}$} & $113 /-45$ & $39.55 /-15.75$ & knee neutral $0^{\circ}$ \\
\cline { 2 - 4 } & $90 /-30$ & $31.50 /-10.5$ & knee flex $90^{\circ}$ \\
\hline \multirow{4}{*}{ Knee flexion } & 113 (stand) & 39.55 & \\
& 125 (prone) & 43.75 & hip neutral $0^{\circ}$ \\
& 159 (knee) & 55.65 & \\
\cline { 2 - 4 } & 80 (stand) & 28.00 & hip flex $90^{\circ}$ \\
\hline Ankle plantarflexion & $38 /-35$ & $13.30 /-12.25$ & knee neutral $0^{\circ}$ \\
\cline { 2 - 4 } dorsiflexion & $36 /-33$ & $12.60 /-11.55$ & knee flex $90^{\circ}$ \\
\hline
\end{tabular}

\subsection{The trajectory planning}

Seeking the joint trajectories of the human leg is a wide research problem. In this article, the proposed method is based on the fifth degree polynomial. One of the advantages of this 
polynomial is that the velocity and acceleration at the beginning and at the end of motion is zero. To start, it is necessary to determine the function for each natural coordinate in the initial position for the moment in time $t_{0}$ and end at the time $t_{k}$. By using the fifth degree polynomial, it is essential to plan the velocity and acceleration at the beginning and the end of the movement. The fifth degree polynomial takes the form

$$
\theta(t)=s_{0}+s_{1} t+s_{2} t^{2}+s_{3} t^{3}+s_{4} t^{4}+s_{5} t^{5}
$$

with restrictions

$$
\begin{array}{lll}
\theta(0)=\theta_{p} & \theta\left(t_{k}\right)=\theta_{k} & \dot{\theta}(0)=\dot{\theta}_{p} \\
\dot{\theta}\left(t_{k}\right)=\dot{\theta}_{k} & \ddot{\theta}(0)=\ddot{\theta}_{p} & \ddot{\theta}\left(t_{k}\right)=\ddot{\theta}_{k}
\end{array}
$$

then we receive

$$
\begin{array}{ll}
\theta_{p}=s_{0} & \theta_{k}=s_{0}+s_{1} t_{k}+s_{2} t_{k}^{2}+s_{3} t_{k}^{3}+s_{4} t_{k}^{4}+s_{5} t_{k}^{5} \\
\dot{\theta}_{p}=s_{1} & \dot{\theta}_{k}=s_{1}+2 s_{2} t_{k}+3 s_{3} t_{k}^{2}+4 s_{4} t_{k}^{3}+5 s_{5} t_{k}^{4} \\
\ddot{\theta}_{p}=2 s_{2} & \ddot{\theta}_{k}=2 s_{2}+6 s_{3} t_{k}+12 s_{4} t_{k}^{2}+20 s_{5} t_{k}^{3}
\end{array}
$$

where the final formula takes form of

$$
\left[\begin{array}{l}
s_{0} \\
s_{1} \\
s_{2} \\
s_{3} \\
s_{4} \\
s_{5}
\end{array}\right]=\left[\begin{array}{cccccc}
1 & t_{0} & t_{0}^{2} & t_{0}^{3} & t_{0}^{4} & t_{0}^{5} \\
0 & 1 & 2 t_{0} & 3 t_{0}^{2} & 4 t_{0}^{3} & 5 t_{0}^{4} \\
0 & 0 & 2 & 6 t_{0} & 12 t_{0}^{2} & 20 t_{0}^{3} \\
1 & t_{k} & t_{k}^{2} & t_{k}^{3} & t_{k}^{4} & t_{k}^{5} \\
0 & 1 & 2 t_{k} & 3 t_{k}^{2} & 4 t_{k}^{3} & 5 t_{k}^{4} \\
0 & 0 & 2 & 6 t_{k} & 12 t_{k}^{2} & 20 t_{k}^{3}
\end{array}\right]^{-1}\left[\begin{array}{c}
\theta_{0} \\
\dot{\theta}_{0} \\
\ddot{\theta}_{0} \\
\theta_{k} \\
\dot{\theta}_{k} \\
\ddot{\theta}_{k}
\end{array}\right]
$$

\subsection{Forward kinematics and the workspace}

By using the forward kinematics, it is possible to determine the position and orientation of the end effector. There are several methods to resolve this problem from geometrical to analytical by using homogeneous transformation matrices method and Denavit-Hartenberg's systematic representation of reference systems (Głowiński et al., 2015). Our kinematic model of the leg is in the sagittal plane, then one can easily extract its direct kinematics parameters

$$
\begin{aligned}
& x_{O}=l_{t} \cos \theta_{h}+l_{l} \cos \left(\theta_{h}-\theta_{k}\right)-l_{f} \sin \left(\theta_{h}-\theta_{k}-\theta_{a}\right) \\
& y_{O}=l_{t} \sin \theta_{h}+l_{l} \sin \left(\theta_{h}-\theta_{k}\right)+l_{f} \cos \left(\theta_{h}-\theta_{k}-\theta_{a}\right) \\
& r=\sqrt{x_{O}^{2}+y_{O}^{2}} \quad r_{1}=\sqrt{l_{t}^{2}+l_{l}^{2}+2 l_{t} l_{l} \cos \theta_{k}} \quad r_{2}=\sqrt{l_{l}^{2}+l_{f}^{2}+2 l_{l} l_{f} \sin \theta_{a}} \\
& \theta_{k}=\alpha_{l}-\beta_{l} \quad \theta_{a}=\gamma_{l}-\beta_{l}-\frac{\pi}{2} \quad \theta_{f}=\pi-\gamma_{l}
\end{aligned}
$$

The workspace is an important performance index of a human leg in the rehabilitation process. This workspace can be divided into two categories: the position workspace and the orientation angle workspace. The position workspace indicates the region reached by the reference point on the end-effector. The orientation angle workspace indicates a set of angle ranges by which the end-effector can reach with certain orientation for any point within the reachable position workspace. The workspace coordinates of the human leg including $n$-joints constraints can be obtained by using formulas

$$
x=\sum_{i=1}^{n} l_{i} \cos \left(\sum_{p=1}^{i} \theta_{p}\right) \quad y=\sum_{i=1}^{n} l_{i} \sin \left(\sum_{p=1}^{i} \theta_{p}\right)
$$


Figure 3 shows the leg workspace in the sagittal plane of a $1.75 \mathrm{~m}$ height person, with joint constraints. This workspace is characterized in a half cross-section by singular curves. The workspace topology is defined by the number of cusps and nodes that appear on these singular curves.

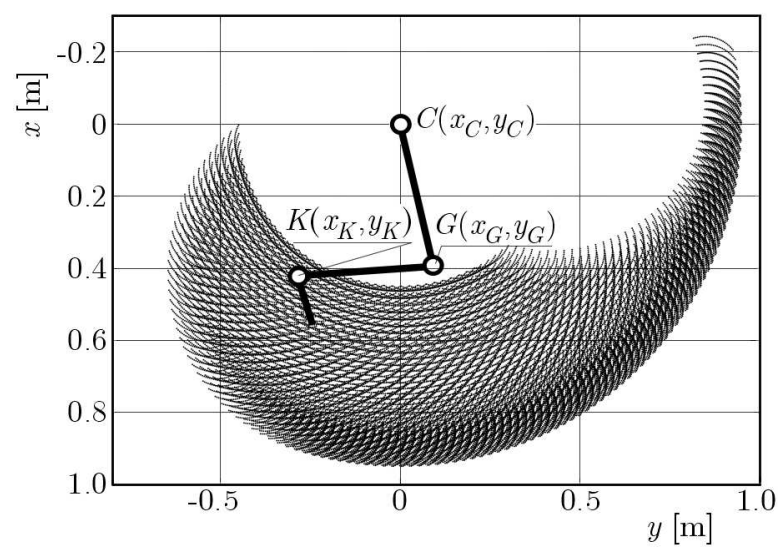

Fig. 3. The human leg workspace for the constrained optimization problem

\section{Algorithm approach to solving the inverse kinematics and obtaining the trajectory of the human leg}

In inverse kinematics we want to find the set of joint angles that produce a specific end-effector position. If we have a configuration of our model, and we want to move it to a new position, then, we want to compute the change in the joint angles needed to produce the change in endpoint position. In Fig. 2a it is assumed that it is not straightforward to obtain the inverse kinematics of a simple 3 -joint leg model. If we know the orientation (e.g. the foot angle $\theta_{f}$ ) and the final position, it is possible to obtain analytical solutions by using formulas

$$
\begin{aligned}
& \theta_{k}=\arccos \frac{\left(x_{O}-l_{f} \sin \theta_{f}\right)^{2}+\left(y_{O}-l_{f} \cos \theta_{f}\right)^{2}-l_{t}^{2}-l_{l}^{2}}{2 l_{t} l_{l}} \\
& \theta_{h}=\arctan \frac{y_{O}-l_{f} \cos \theta_{f}}{x_{O}-l_{f} \sin \theta_{f}}+\arccos \frac{l_{t}^{2}-l_{l}^{2}+\left(x_{O}-l_{f} \sin \theta_{f}\right)^{2}+\left(y_{O}-l_{f} \cos \theta_{f}\right)^{2}}{2 l_{t} \sqrt{\left(x_{O}-l_{f} \sin \theta_{f}\right)^{2}+\left(y_{O}-l_{f} \cos \theta_{f}\right)^{2}}} \\
& \theta_{a}=\theta_{h}-\theta_{k}-\theta_{f}+\frac{\pi}{2}
\end{aligned}
$$

If we do not know the orientation, there is an infinite number of solutions. Thus, the numerical methods appears to be acceptable.

\subsection{Formulation of the optimization problem}

When dealing with a redundant manipulator, as the proposed leg model has more degrees of freedom than necessary to perform a certain task, the remaining degrees of freedom give a set of feasible solutions of the inverse kinematics. Among these solutions, it is recommended to choose thst satisfying a certain criterion. The main goal is to find the compromising solutions between several criteria. The criteria can be formulated as ROM and the distance between the goal and end the effector. One of the criteria used in inverse kinematics algorithms is to restrict joint limits. This can be done by optimizing a potential function with very high values in the neighbourhood of a limit. This function can be expressed as

$$
w(\theta)=\frac{1}{2 n} \sum_{i=1}^{n} \frac{\left(\theta_{i, \max }-\theta_{i, \min }\right)^{2}}{\left(\theta_{i, \max }-\theta_{i}\right)\left(\theta_{i}-\theta_{i, \min }\right)}
$$


where $n$ is the number of joints. This function gives, as we can see in Fig. 4, a very high potential when approaching the knee joint limit, and the minimum value at the midpoint.

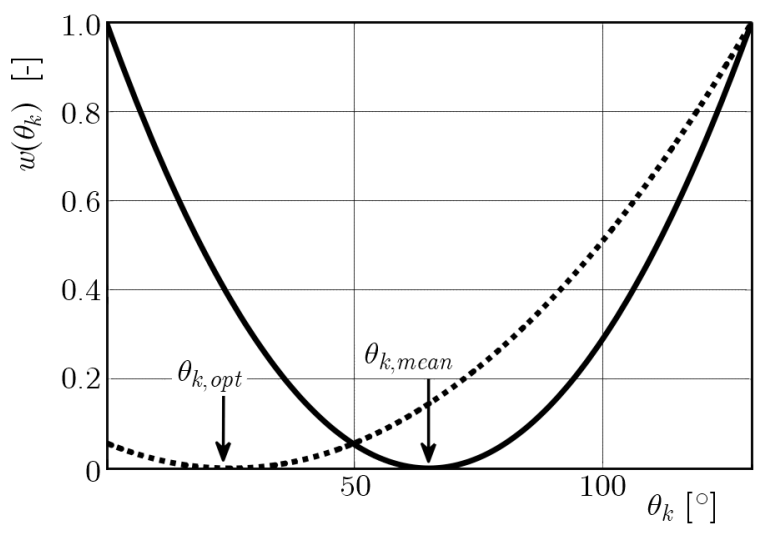

Fig. 4. The rise of the potential function when approaching knee joint limits

The next criterion can be formulated as a distance, where $O\left(x_{O}, y_{O}\right)$ represents the goal

$$
\begin{aligned}
& x_{O}-\left[l_{t} \cos \theta_{h}+l_{l} \cos \left(\theta_{h}-\theta_{k}\right)-l_{f} \sin \left(\theta_{h}-\theta_{k}-\theta_{a}\right)\right]<0.0001 \\
& y_{O}-\left[l_{t} \sin \theta_{h}+l_{l} \sin \left(\theta_{h}-\theta_{k}\right)+l_{f} \cos \left(\theta_{h}-\theta_{k}-\theta_{a}\right)\right]<0.0001
\end{aligned}
$$

A general formulation of the optimization problem would be

$$
\min _{\theta} f(\theta) \quad \text { such that } \quad c(\theta)<0 \text { and } \quad c_{e q}(\theta)=0
$$

In formulation (4.4) $\theta$ is the vector of optimization variables, $c$ and $c_{e q}$ are vectorial functions involved in the inequality and equality constraints, respectively. The optimum angles of joints are defined as $\theta_{h, o p t}, \theta_{k, o p t}, \theta_{a, o p t}$. They can be personalized for each person. Problems without any constraint $c$ and $c_{e q}$ are called unconstrained while the others are constrained. The objective function $f(\theta)$ should be minimized and it is based on the comfort zone of every joint, and can be expressed as

$$
f(\theta)=\left(\frac{\theta_{h}-\theta_{h, o p t}}{\theta_{h, \min }-\theta_{h, \max }}\right)^{2}+\left(\frac{\theta_{k}-\theta_{k, \text { opt }}}{\theta_{k, \min }-\theta_{k, \max }}\right)^{2}+\left(\frac{\theta_{a}-\theta_{a, o p t}}{\theta_{a, \min }-\theta_{a, \max }}\right)^{2}
$$

By using Matlab package, it is possible to use different solvers depending on the objective function and constrains. In his problem, the constrains are nonlinear and the objective function is quadratic, then the best fit solver is fmincon.

\subsection{Inverse kinematics algorithm}

In Fig. 5, the inverse kinematics algorithm is illustrated. The algorithm is divided into four steps. The first step begins by initialization. It is necessary to determine the height $H$ of a subject and calculate length of thigh $l_{t}$, shank $l_{l}$ and foot $l_{f}$ as the height function. The next part of this step is the determination of the initial hip, knee and ankle angles, respectively, $\theta_{h}$, $\theta_{k}, \theta_{a}$, and calculation of the initial effector position by using forward kinematics (3.1). Next, with joints constraints and formulas (3.2) the workspace and the comfort zone of each joint should be designated. In the second step, the end position coordinates should be given. The program checks whether the final coordinates are in the workspace. If not, it is necessary to find new coordinates. The third steps of the optimization begins by using Matlab package fmincon solver. The solution is a matrix with three angles in the final position. The angles are the most comfortable with taking into account the comfort zone of each joint. If the result is not satisfied, 
the next optimization should be done, or the comfort zone calculated properly. In the fourth step, the maximum velocity, acceleration and the minimum time of movement is determined. After that step, the trajectory by using fifth degree polynomial (2.4) can be obtained.

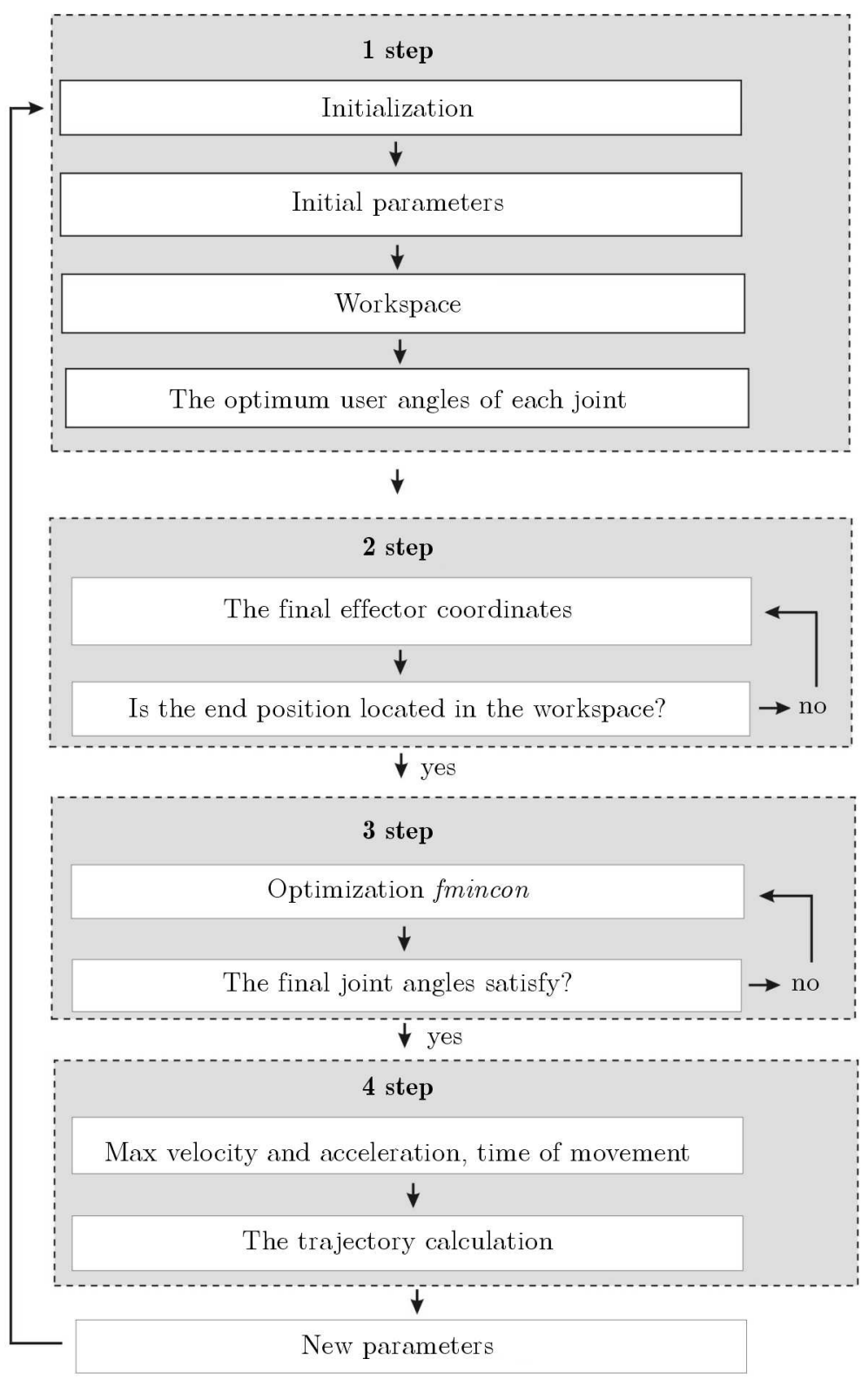

Fig. 5. The inverse kinematics algorithm

\section{Results}

For a $1.75 \mathrm{~m}$ person height, the initial angles are obtained as $\theta_{h i}=86^{\circ}, \theta_{k i}=17^{\circ}, \theta_{a i}=-6^{\circ}$. From forward kinematics (3.1), the end-effector coordinates are calculated as $A\left(x_{O}=0.089\right.$, $\left.y_{O}=0.856\right)$. If we know the final point as $C\left(x_{f}=0.4, y_{f}=-0.4\right)$, based on the provided algorithm and the determined optimal angles, the final angles are calculated as $\theta_{h f}=17^{\circ}$, $\theta_{k f}=108^{\circ}, \theta_{a f}=-6^{\circ}$. Then by using fifth degree polynomial (2.2), the trajectory can be determined. Figure 6 shows the visualization and the angles, angular velocity and acceleration of each joint. The time of motion is $2 \mathrm{~s}$. The maximum angular knee joint velocity is about $80^{\circ} / \mathrm{s}$, whereas acceleration $140^{\circ} / \mathrm{s}^{2}$. It is acceptable from the biomechanical point of view (Głowiński 
et al., 2015). The generated result shows high similarity between the model motion and the real human leg motion.

(a)

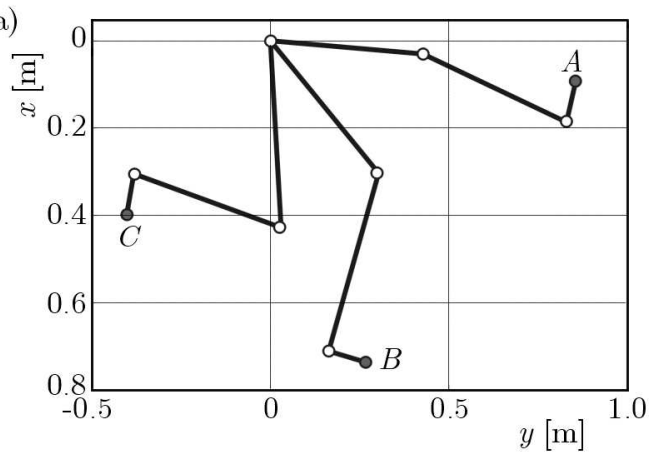

(c)

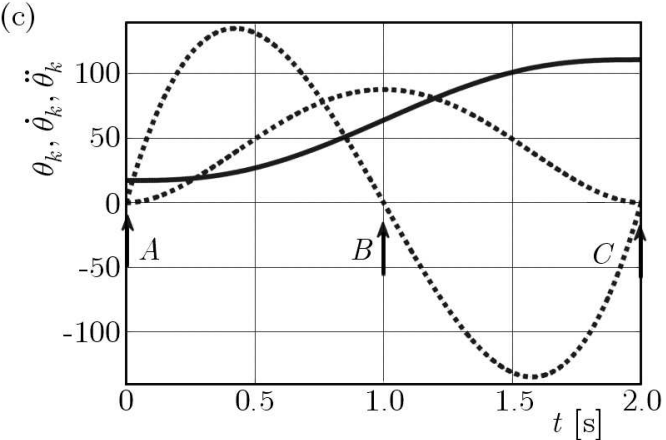

(b)

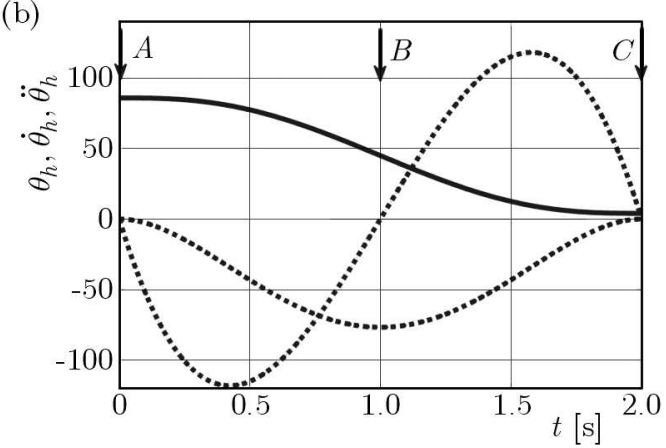

(d)

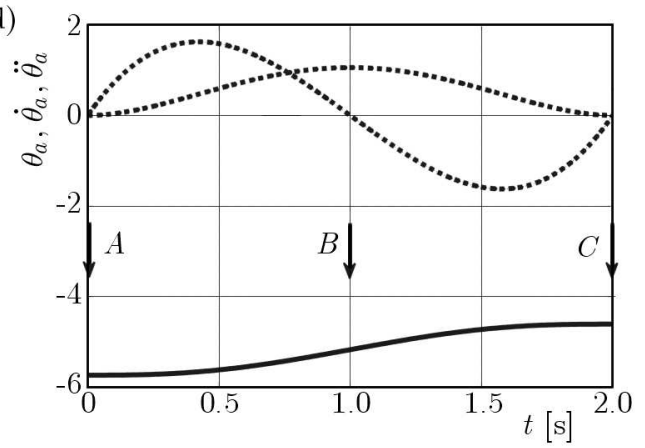

Fig. 6. Graph presenting displacement, angular velocity and acceleration for the fifth degree polynomial describing motion of the human leg between the two positions, human leg model displacement (a), hip (b), knee (c), foot (d)

From Fig. 6, it is observed that the final leg position is close to the target points $C\left(x_{f}=0.4, y_{f}=-0.4\right)$. The proposed methodology has been validated for different starting points and the results satisfied the criteria. Repeatability is a significant issue in our algorithm. It is very important for the user that the Matlab solver behaves consistently and is not sensitive to changes in the starting point.

\section{Conclusion}

In this paper, an approach for modelling and simulation of the human leg inverse kinematics is presented. When planning the trajectory of the human leg, which will be used for rehabilitation, individual patient capabilities need to be taken into consideration. This can be done by a preliminary study. Subsequently, the physician selects exercises depending on disease. It is particularly important after stroke with spasticity. As mentioned earlier, it should be noted that this study is limited to analysis of movements in the sagittal plane. Further investigations are thus needed in order to generalize our findings to other planes.

When planning the trajectory, significant simplifications are being made by assuming the maximum acceleration values for each degree of freedom. If the maximum acceleration values are improperly selected, this can lead to the possibility of exceeding human joint limits. From the presented simulation results, the best method for path planning is a fifth degree polynomial. According to the simulation results, it is decided to improve the mechanical construction. In real situations, for particular real exercises, there are much more parameters needed to be considered in the modelling, for example, the inserted force or the stability criterion. Further experiments are to be carried out in order to verify the modelling results in experiments. 


\section{References}

1. Chaffin D., Andersson G., 1991, Occupational Biomechanics, 2nd edn., John Wiley \& Sons

2. GŁowiński S., Krzyżyński T., Pecolt S., Maciejewski I., 2015, Kinematics and dynamics of an exoskeleton arm, Archives of Applied Mechanics, 85, 75-87, DOI 10.1007/s00419-014-0900-8

3. Gu E.Y.L., 2013, A Journey from Robot to Digital Human, Mathematical Principles and Applications with MATLAB Programming, Springer

4. Nocedal J., Wright S.J., 1999, Numerical Optimization, Springer

5. Parker J.A., Khoogar A.R., Goldberg D.E., 1989, Inverse kinematics of redundant robots using genetic algorithms, Proceedings, IEEE International Conference on Robotics and Automation, 1, 271-276

6. Porta J.M., Ros L., Thomas F., 2005, Inverse kinematic solution of robot manipulators using interval analysis, 4th International Workshop on Computational Kinematics

7. Rao R.S., Asaithambi A., Agrawal S.K., 1998, Inverse kinematic solution of robot manipulators using interval analysis, Journal of Mechanical Design, 120, 1, 147-150

8. Tejomurtula S., KaK S., 1999, Inverse kinematics in robotics using neural networks, Information Sciences, 116, 2/4, 147-164

9. http://philschatz.com/anatomy-book/contents/m46398.html, http://creativecommons.org/licenses/by/4.0/ Anatomy and Physiology Textbook (access 10.02.2015) 